\title{
The effects of ranibizumab injections on fluorescein angiographic findings and visual acuity recovery in age-related macular degeneration
}

This article was published in the following Dove Press journal:

Clinical Ophthalmology

19 May 2014

Number of times this article has been viewed

\author{
Hulya Gungel' \\ Ozen Ayranci \\ Osmanbasoglu' \\ Cigdem Altan ${ }^{2}$ \\ Deniz Oygar Baylancicek ${ }^{3}$ \\ Isil Basgil Pasaoglu² \\ IIstanbul Education and Research \\ Hospital, Eye Clinic, ${ }^{2}$ Beyoglu Eye \\ Education and Research Hospital, \\ ${ }^{3}$ Kudret Eye Hospital, Istanbul, Turkey
}

Correspondence: Hulya Gungel Istanbul Education and Research Hospital, Eye Clinic, Kasap İlyas Mh Org Abdurrahman Nafiz Gürman Cd PK: 34098, Fatih, Istanbul,Turkey Tel +905325482088 Fax +902126320060 Email hulyagungel@msn.com
Aim: The objective of the study reported here was to evaluate the effect of ranibizumab on retinal circulation times and vessel caliber and to analyze the correlation of these factors with visual acuity (VA) prognosis in patients with age-related macular degeneration (AMD).

Subjects and methods: This prospective cohort study included 52 eyes of 46 patients (mean age 73.5 years [standard deviation 7.7]; 28 males, 18 females). The study parameters were best-corrected visual acuity (BCVA), central macular thickness (CMT) (pre- and posttreatment: for 3 months after the last injection), retinal circulation times, diameter of retinal arteriole (DRA), and diameter of retinal vein (DRV) (pre- and posttreatment: after a loading dose of three consecutive injections of ranibizumab with a 4-week interval in the initial phase). The pretreatment, posttreatment measurements, and their differences were recorded for analyses. The injections were repeated when needed. Eyes were grouped into one of two groups according to VA recovery: Group 1, cases showing significant recovery of VA $(n=21,37 \%)$, and Group 2, cases showing preservation of VA $(n=22,42 \%)$ and deterioration of VA $(n=11,21 \%)$. Differences were compared statistically in and between groups. Logistic regression analysis was undertaken to determine the correlation of these parameters with VA recovery.

Results: There was a significant reduction in DRA $(P=0.007)$ and CMT levels $(P<0.001)$ in both study groups after treatment. When the two groups were compared, the differences in pretreatment values of DRA $(P=0.001)$, DRV $(P=0.017)$, CMT $(P=0.039)$, and mean BCVA $(P=0.00)$ were found to be statistically significant. Posttreatment changes in DRA $(P=0.013)$ and mean CMT $(P=0.010)$ were found to be factors related to VA recovery by logistic regression analysis.

Conclusion: Our findings reveal that ranibizumab treatment is associated with decrease in DRA, CMT, and significant improvement in VA recovery. Further, taking into account the cases in which VA was preserved, when needed, ranibizumab should be re-injected after the loading dose.

Keywords: vessel caliber, prognosis, central macular thickness, retinal vein diameter, retinal arteriole diameter

\section{Introduction}

Neovascular age-related macular degeneration (AMD), a leading cause of severe visual loss in elderly persons 60 years and older in industrialized countries, is an ocular disease involving angiogenesis and is thus particularly destructive to central visual acuity (VA).

The development of choroidal neovascularization (CNV) in AMD has been stated to be induced by various molecular regulators, including vascular endothelial growth factor (VEGF), fibroblast growth factor 2, pigment epithelium-derived growth factor, angiopoietins, and extracellular matrix factors. ${ }^{2}$ VEGF is expressed at 
increased concentrations in the setting of macular edema, and VEGF levels in humans are likely to be correlated to the degree of the activation of neovascularization and vascular permeability. ${ }^{3}$ Accordingly, in the last few years, the intraocular administration of ranibizumab (Lucentis; Genentech, Inc., San Francisco, CA, USA) to suppress VEGF has been proven effective in preserving and, in some cases, restoring vision in eyes with neovascular AMD. ${ }^{4-7}$

Changes in retinal vascular caliber may reflect changes in retinal blood flow, inflammation, ischemia, and endothelial function. ${ }^{8,9}$ Thus, measurement of retinal vascular caliber has been suggested to provide a marker of underlying disease severity in patients with neovascular AMD and enable the clarification of factors influencing visual outcome in patients needing anti-VEGF treatment. ${ }^{10}$

Therefore, based on the documented efficacy of antiVEGF agents in the treatment of patients with neovascular $\mathrm{AMD},{ }^{7,8,10,11}$ the study reported here, performed at Beyoglu Eye Education and Research Hospital, was conducted to evaluate the difference in retinal vessel circulation response to treatment with anti-VEGF agents in patients with different VA outcomes. For this reason, we prospectively evaluated the data of 46 patients ( 52 eyes) with neovascular AMD. The vascular response to anti-VEGF treatment was evaluated by retinal vessel caliber and circulation-time measurements using fluorescein angiography (FA) before and after treatment. The correlations between the vascular parameters and change in VA and central macular thickness (CMT) were evaluated statistically.

\section{Subjects and methods Subjects}

Fifty-two eyes of 46 consecutive patients with newly diagnosed neovascular AMD were included in this prospective study. The two inclusion criteria were: (1) age of 50 years or more, and (2) presence of a lesion sized $\leq 2$ disc diameters as the greatest linear dimension and subfoveal active CNV with scar formation $<50 \%$ of the total lesion size, as demonstrated on FA. No restriction to the type of the membrane was made. Patients with coexisting disease such as uncontrolled hypertension, diabetes mellitus, retinal vascular occlusion, uveitis, trauma, myopia of more than 6 diopters, or vitreomacular interface disorders such as epiretinal membrane were excluded. Patients who had a pigment epithelial rupture, had previous vitreoretinal surgery, or received photodynamic therapy or intravitreal injections were also excluded.

Written informed consent was obtained from each subject following a detailed explanation of the objectives and study protocol, which was conducted in accordance with the ethical principles stated in the Declaration of Helsinki and approved by the Beyoglu Eye Education and Research Hospital's institutional ethics committee.

\section{Examinations and treatment protocol}

All patients underwent a complete ophthalmic examination at each visit, including assessment of BCVA using standardized refraction with a Snellen chart, biomicroscopic evaluation, intraocular-pressure measurement with an applanation tonometer, and funduscopic examination. Optical coherence tomography (OCT) images were taken at pretreatment examination and at every visit. FA was recorded during the pretreatment examination, when activation or new neovascularization was suspected, and at 4-6 weeks after the third ranibizumab injection as a posttreatment parameter. All patients received a loading dose of three consecutive injections of ranibizumab at 4-week intervals in the initial phase. Patients were then followed monthly by clinical and OCT examinations. If recurrence was detected, retreatment was performed with a single ranibizumab injection on an as-needed basis. Retreatment criteria were defined as the presence of one of the following: increase in CMT of $\geq 100$ microns or increase in CMT even if $<100$ microns when VA deteriorated (VA reduction of at least five letters), presence of intraretinal or subretinal fluid on OCT, newly developed intra- or subretinal hemorrhage on ophthalmoscopy, or findings of an active lesion during FA.

\section{Study parameters}

The study parameters were age, injection number, pre- and posttreatment BCVA, CMT, and FA parameters (retinal circulation times, diameters of retinal arteriole and vein). BCVA was measured with a Snellen chart and converted to logarithm of the minimum angle of resolution (logMAR) units for analysis. The differences in study parameters were computed by subtracting the pretreatment values from the posttreatment values. The difference between mean pre- and posttreatment BCVA measurements was accepted as recovery of VA. Significant recovery of VA was defined as an improvement of $0.2 \log$ MAR units or more, preservation of vision was defined as VA within two lines of pretreatment, and deterioration of VA was considered to be a reduction of at least two lines. Eyes were identified according to this classification.

Patients were divided into two groups: Group 1 comprised cases in which significant visual recovery was identified, and Group 2 comprised all others. When stabilization of VA was achieved (52 eyes) and/or the fluid-free OCT images were obtained (44 eyes) 3 months after the last injection, the BCVA and CMT were recorded and accepted as posttreatment 
values. Retinal circulation times and vessel calibers were assessed as angiographic parameters and were recorded at the pretreatment examination and also at 4-6 weeks after the third ranibizumab injection as posttreatment parameters.

\section{Angiographic evaluation}

Angiographic examinations were undertaken using a Heidelberg Retinal Angiogram-2 (HRA-2; Heidelberg Engineering $\mathrm{GmBH}$, Heidelburg, Germany) and parameters were measured from the recorded FA images. When both eyes of the same patient were included in the study, each eye was treated on an as-needed basis according to the re-injection criteria. If both eyes had active disease at the same time, FA examinations were done separately for each eye. If one eye had inactive disease and the other had active disease, we began capturing the images from the eye with active disease. Five parameters on FA for each eye were studied by two retina specialists (who were both masked to the findings of the patients) at the same time, while one specialist measured the parameters. The FA parameters included arm-toretina circulation time (ARC, seconds), arteriovenous transit time (AVT, seconds), venous filling time (VFT, seconds), diameter of retinal vein (DRV, $\mathrm{mm}$ ), and diameter of retinal arterioles (DRA, mm). The ARC was recorded as the time from the onset of dye injection to its first appearance in the central retinal artery. The AVT was defined as the length of time between the first appearance of dye in the retinal arteries to the first appearance of laminar flow in the vein. The VFT was recorded from the time when venous laminar blood flow began to appear to the time when the retinal vein was completely filled and appeared brighter than the artery.

Vessel diameter measurements were performed using one of the quantitative analysis tools available in the HRA-2 software, Heidelberg Eye Explorer (v 1.8.6.0). The measurement point was between 1 and 2 disc diameters from the optic disc edge before any bifurcation and arteriovenous crossing of the vessel. As there can be an arteriovenous crossing and branching difference between subjects and even between quadrants in the same eye, the measurements from each quadrant may not be taken, so only one quadrant was used for evaluation. The inferior temporal quadrant was mostly used for this purpose, and repeated measurements were taken from the exact same localization as the first measurement. If there was arteriovenous winding or crossing at the measurement site, the vessel at the superior temporal quadrant was evaluated. The measurements were taken from wall to wall as a fluorescein-filled column, when the vessel was fully filled with dye. Three different measurements were taken at

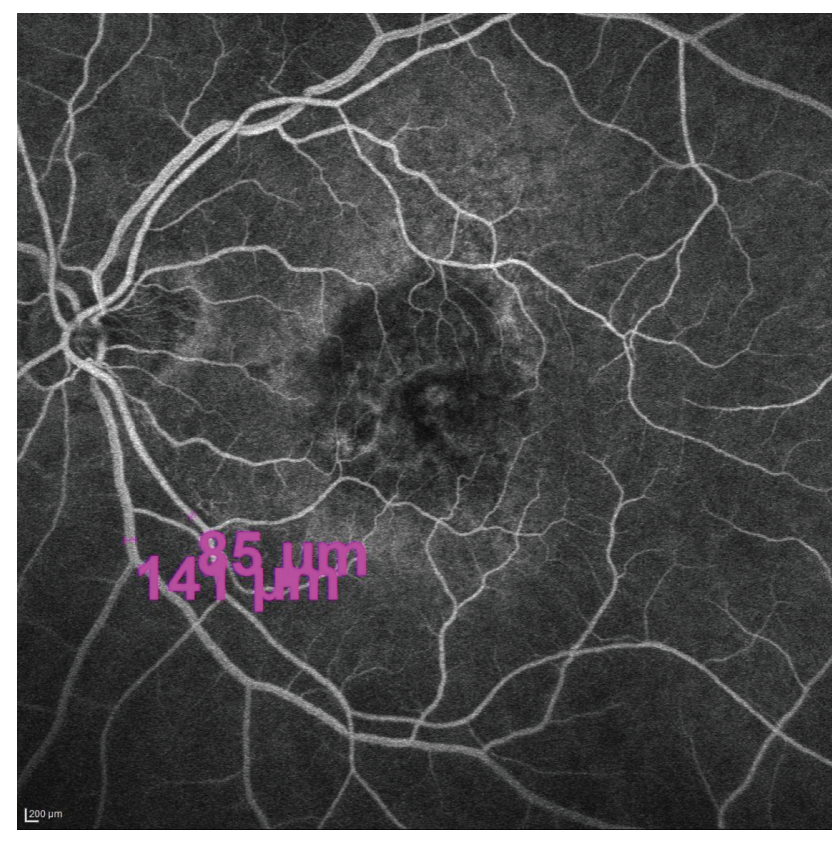

Figure I Diameter measurement of retinal artery and vein.

the same localization on the vessel wall in the same session (Figure 1). If the difference between measurements was less than $0.002 \mathrm{~mm}$, all three measurements were averaged.

\section{Macular thickness}

OCT images were obtained using a Stratus OCT instrument (OCT3; Carl Zeiss Meditech Inc, Dublin, CA, USA). Fast macular scan mode was used and the central $1.0 \mathrm{~mm}$ retinal thickness was calculated automatically by retinal map analysis.

\section{Intravitreal anti-VEGF injections}

Intravitreal injections of anti-VEGF $(0.5 \mathrm{mg} / 0.1 \mathrm{~mL})$ (Lucentis; Genentech, Inc) were performed under sterile conditions as described elsewhere. ${ }^{11}$

\section{Outcomes}

To evaluate ranibizumab-induced changes, the pretreatment and posttreatment CMT and VA values and angiographic parameters were analyzed in the overall study population.

The correlation between VA recovery and age, total injection number, CMT, pretreatment angiographic values, and their differences after treatment were analyzed in the overall study population.

The study parameters (age; injection number; pretreatment values; and differences of BCVA, CMT, and angiographic parameters) were compared statistically between the two groups in terms of VA recovery. Logistic regression 
was applied to detect the probable predictive parameters for VA prognosis after three ranibizumab injections.

\section{Statistical analysis}

Data are expressed using the statistical mean and standard deviation (SD). Statistical analysis was performed using SPSS software (v 20.0; IBM, Armonk, NY, USA). The distribution of the variables was checked against the reference distribution using the Kolmogorov-Smirnov test. In the evaluation of the quantitative data, the independent samples (unpaired) $t$-test and pair-wise $t$-test (for repeated measurements) were used. Continuous variables were analyzed via Student's $t$-test for normally distributed variables, while nonparametric tests were used for parameters that were not normally distributed. Where appropriate, Pearson's correlation test and logistic regression were used to evaluate the relationship between VA recovery and each parameter. A $P$ value $<0.05$ was considered statistically significant.

\section{Results}

\section{Patient demographics and injection number}

A total of 52 eyes were included in the study. The majority of the patients were male (28 versus 18 ) and the mean age of the study population was 73.7 (SD 7.7) years. Twenty-four eyes had three injections, two eyes had nine injections, and the mean injection number was 4.25 (SD 1.73) in the overall study population.

Posttreatment BCVA and OCT parameters were evaluated 11.25 (SD 6.04) months (7-27 months) after the pretreatment visit.

\section{Outcome measures}

Table 1 shows the comparison of pretreatment and posttreatment BCVA, CMT, and angiographic parameters in the overall study population. While there was not a significant difference in BCVA between pretreatment and posttreatment values, there was a significant thinning of the CMT with the treatment of ranibizumab in the overall study population $(P<0.001)$.

According to the pretreatment values, DRA was the only FA-related parameter to decrease significantly $(P=0.007)$ after treatment.

When we analyzed the correlation of VA recovery with the pretreatment study parameters in the overall study population, the correlations between VA recovery and age, injection number, and retinal circulation time were found to be insignificant, but significant correlations between VA recovery and pretreatment BCVA $(r=-0.620, P=0.000)$, pretreatment DRV $(r=-0.349, P=0.013)$, and pretreatment DRA ( $r=-0.284, P=0.048)$ were detected.

The correlation between VA recovery and differences between pre- and posttreatment values of the study parameters were also analyzed, and only the difference of DRA ( $r=-0.336, P=0.022$ ), using Pearson's correlation test (Table 2), was found to be significant. Pretreatment BCVA was found to be highly correlated with posttreatment BCVA ( $r=0.433, P=0.001)$, difference of DRA $(r=0.402, P=0.006)$, pretreatment CMT $(r=0.334, P=0.016)$, and pretreatment $\operatorname{DRV}(r=0.350, P=0.013)$.

Although there was no significant difference between pretreatment and posttreatment BCVA in the overall study population, significant VA recovery was detected in $19(37 \%)$ eyes (Group 1), and preservation of VA was detected in $22(42 \%)$ eyes and deterioration of VA was detected in eleven $(21 \%)$ eyes of Group $2(n=33)$ participants. Mean VA change was -0.61 (SD 0.30) logMAR units in Group 1 and 0.17 (SD 0.30) logMAR units in Group 2.

When the two groups were compared in terms of study parameters, the differences in pretreatment DRA $(P=0.001)$, pretreatment DRV $(P=0.017)$, pretreatment $\mathrm{CMT}(P=0.039)$, and pretreatment BCVA $(P<0.001)$ between groups were

Table I Comparison of pre- and posttreatment values in the study population

\begin{tabular}{llll}
\hline Study parameter & Pretreatment, mean (SD) & Posttreatment, mean (SD) & P-value* \\
\hline ARC (seconds) & $16.70(4.28)$ & $17.44(5.10)$ & 0.327 \\
AVT (seconds) & $2.30(1.02)$ & $2.16(0.92)$ & 0.453 \\
VFT (seconds) & $11.69(2.70)$ & $11.85(2.88)$ & 0.688 \\
DRV (mm) & $0.14(0.02)$ & $0.14(0.03)$ & 0.852 \\
DRA (mm) & $0.11(0.03)$ & $0.10(0.02)$ & $0.007^{\dagger}$ \\
Mean CMT ( $\mu$ m) & $309.10(90.33)$ & $226.00(58.22)$ & $0.000^{\dagger}$ \\
Mean BCVA (logMAR unit) & $0.76(0.48)$ & $0.64(0.43)$ & 0.074 \\
\hline
\end{tabular}

Notes: *Paired samples $t$-test; ${ }^{\dagger} P<0.05$.

Abbreviations: ARC, arm-retina circulation; AVT, arteriovenous transit time; BCVA, best-corrected visual acuity; CMT, central macular thickness; DRA, diameter of retinal artery; DRV, diameter of retinal vein; logMAR, logarithm of the minimum angle of resolution; SD, standard deviation; VFT, venous filling time. 
Table 2 Analysis of correlations between visual acuity recovery and study parameters in the study population

\begin{tabular}{lllll}
\hline Parameter & $P$-value* & $\begin{array}{l}\text { Pretreatment } \\
\text { value }\end{array}$ & P-value* & $\begin{array}{l}\text { Pre-posttreatment } \\
\text { differences of value }\end{array}$ \\
\hline BCVA & $r=-0.620$ & 0.000 & $r=0.432$ & 0.001 \\
CMT & $r=-0.127$ & 0.375 & $r=-0.245$ & 0.090 \\
DRA & $r=-0.284$ & 0.048 & $r=-0.336$ & 0.022 \\
DRV & $r=-0.349$ & 0.013 & $r=-0.141$ & 0.351 \\
ARC & $r=-0.044$ & 0.800 & $r=-0.068$ & 0.741 \\
AVT & $r=0.097$ & 0.568 & $r=0.274$ & 0.0167 \\
VFT & $r=0.084$ & 0.601 & $r=-0.030$ & 0.741 \\
Age & $r=-0.062$ & 0.710 & & \\
TIN & $r=0.200$ & 0.156 & & \\
Note & & &
\end{tabular}

Note: *Pearson correlation test.

Abbreviations: ARC, arm-retina circulation time; AVT, arteriovenous transit time; BCVA, best-corrected visual acuity; CMT, central macular thickness; DRA, diameter of retinal artery; DRV, diameter of retinal vein; TIN, total injection number; VFT, venous filling time.

found to be statistically significant (Table 3). In Group 1, pretreatment DRV and DRA were both larger, CMT was thicker, and pretreatment VA was worse than in Group 2. When the effect of treatment on study parameters was studied in each group separately, we observed that, in Group 1, the circulation times and DRV did not change significantly, but BCVA, DRA, and CMT values changed significantly ( $P=0.00, P=0.003$, and $P=0.00$, respectively). In Group 2, only the difference in mean CMT values was statistically significant. Also, the difference between pretreatment and posttreatment values of DRA $(P=0.013)$, mean CMT $(P=0.010)$, and mean BCVA $(P<0.001)$ was significant between groups. This means that the magnitude of decrease in arteriole diameter in Group 1 was higher than in Group 2. Further, greater improvement was achieved in terms of CMT and VA in Group 1 (Table 4).

The pre- and posttreatment differences in ARC, AVT, VFT, and DRV in the two groups after treatment were not found to be a significant factor for prediction of VA recovery in a univariate model. Only the changes in DRA and mean CMT values after treatment were significant for the prediction of $\mathrm{VA}$ recovery. This significant effect on the prediction of VA recovery of change in DRA $(P=0.049)$ and mean CMT $(P=0.034)$ was shown in a multivariate model (Table 5$)$.

\section{Discussion}

In this study, we aimed to investigate the effects of ranibizumab on retinal circulation times and vessel caliber and to determine the factors affecting VA recovery in AMD cases. Our findings reveal that ranibizumab treatment is associated with significant retinal arteriolar vasoconstriction, a decrease in CMT, and significant recovery of VA in patients with larger pretreatment retinal vessels and in patients with more prominent reduction in retinal arteriolar diameter and CMT after therapy. No significant changes occurred in overall BCVA scores and retinal circulation times with ranibizumab treatment.

The VA prognosis in patients with CNV due to AMD has changed dramatically within the last few years owing to the introduction of highly effective treatments with anti-VEGF. ${ }^{4,5,12}$ In this respect, we found that ranibizumab treatment was associated with improvement of VA in $37 \%$

Table 3 Pretreatment measurements and comparison of these in Groups I and 2

\begin{tabular}{|c|c|c|c|}
\hline Parameter & $\begin{array}{l}\text { Group I }(n=\mid 9) \text {, } \\
\text { mean (SD) }\end{array}$ & $\begin{array}{l}\text { Group } 2(n=33) \text {, } \\
\text { mean (SD) }\end{array}$ & P-value \\
\hline ARC (seconds) & $17.46(4.59)$ & $16.16(4.42)$ & 0.34 \\
\hline AVT (seconds) & $2.38(0.76)$ & $2.23(1.0)$ & 0.63 \\
\hline VFT (seconds) & 11.92 (3.55) & I I.73 (2.54) & 0.84 \\
\hline DRV (mm) & $0.160(0.22)$ & $0.140(0.02)$ & 0.001 \\
\hline DRA (mm) & $0.116(0.028)$ & $0.099(0.020)$ & 0.017 \\
\hline Mean CMT $(\mu \mathrm{m})$ & 348.89 (108.57) & 294.06 (75.05) & 0.039 \\
\hline Mean BCVA (logMAR) & I.I 7 (0.38) & $0.53(0.37)$ & 0.000 \\
\hline
\end{tabular}

Abbreviations: ARC, arm-retina circulation time; AVT, arteriovenous transit time; BCVA, best-corrected visual acuity; CMT, central macular thickness; DRA, diameter of retinal artery; DRV, diameter of retinal vein; logMAR, logarithm of the minimum angle of resolution; SD, standard deviation; VFT, venous filling time. 
Table 4 The pre-posttreatment differences in study parameters within and between each group

\begin{tabular}{llllll}
\hline Parameter & $\begin{array}{l}\text { Group I (n=19), } \\
\text { mean (SD) }\end{array}$ & P-value* & $\begin{array}{l}\text { Group 2 (n=33), } \\
\text { mean (SD) }\end{array}$ & $\boldsymbol{P}_{\text {-value* }}$ \\
\hline ARC & $-0.25(4.46)$ & 0.80 & $1.34(3.40)$ & 0.12 & 0.282 \\
AVT & $-0.25(1.18)$ & 0.74 & $-0.27(0.79)$ & 0.16 & 0.296 \\
VFT & $-0.25(3.11)$ & 0.82 & $0.30(1.88)$ & 0.44 & 0.646 \\
DRV & $-0.007(0.027)$ & 0.29 & $0.005(0.024)$ & 0.27 & 0.125 \\
DRA & $-0.018(0.019)$ & 0.003 & $-0.003(0.017)$ & 0.35 & 0.013 \\
CMT & $-136.63(104.03)$ & 0.00 & $57.15(93.87)$ & 0.001 & 0.010 \\
BCVA & $-0.61(0.30)$ & 0.00 & $0.17(0.31)$ & 0.03 & 0.000 \\
\hline
\end{tabular}

Notes: *Statistical significance of the difference in measurements in the same group; tstatistical significance of the difference in measurements between two groups.

Abbreviations: ARC, arm-retina circulation time; AVT, arteriovenous transit time; BCVA, best-corrected visual acuity; CMT, central macular thickness; DRA, diameter of retinal artery; DRV, diameter of retinal vein; SD, standard deviation; VFT, venous filling time.

$(n=19)$ of patients, preservation of VA in $42 \%(n=22)$ of patients, and a decrease in VA in $21 \%(n=11)$ of patients at least 4 months after their last injection and with an average of 4.3 injections. The Prospective Optical Coherence Tomography Imaging of Patients with Neovascular Age-Related Macular Degeneration Treated with Intraocular Ranibizumab (PRONTO) study demonstrated that $78 \%$ of patients avoided any loss of letters, $43 \%$ gained at least three lines of vision, and $13.5 \%$ of patients lost five or more letters at their final follow-up visit. ${ }^{13}$ In a recent study by Wickremasinghe et al improvement in VA was reported in 35\% $(n=31)$ and deterioration of VA in $20 \%(n=18)$ of patients at 12 months compared with pretreatment in eyes receiving ranibizumab treatment for neovascular AMD. ${ }^{14}$ Although response to treatment varied substantially among patients, the majority of Comparison of Age-related macular degeneration Treatment Trials (CATT) participants were reported to have the same or improved VA relative to their pretreatment. ${ }^{15}$ Also, some predictors of VA improvement for ranibizumab were identified in the Minimally Classic/Occult Trial of the Anti-VEGF Antibody Ranibizumab in the Treatment of Neovascular Age-related Macular Degeneration (MARINA) study, such as younger age, better pretreatment VA, and smaller CNV area. ${ }^{16}$ Notably, in our study population, there was no significant relation between recovery of VA and age. However, pretreatment VA was determined to have the most important role in recovery of $\mathrm{VA}$, and it was identified that the cases with poor VA before treatment had greater VA improvement.

The association between anti-VEGF treatment and change of retinal vessel caliber has already been reported for patients with AMD. ${ }^{7,10,11,14,17}$ Fontaine et al reported a significant decrease in arteriolar diameter of $4.3 \%, 5$ weeks after the third bevacizumab injection, compared with pretreatment. ${ }^{17}$ Papadopoulou et al also reported a significant constriction of retinal arterioles of $17.6 \%$ at least 7 weeks after the third injection of ranibizumab. ${ }^{7}$ Significant vasoconstriction

Table 5 Predictive factors for the recovery of visual acuity after intravitreal ranibizumab treatment with logistic regression analysis

\begin{tabular}{|c|c|c|c|c|c|}
\hline & & \multirow[t]{2}{*}{$P$-value } & \multirow[t]{2}{*}{ OR } & \multicolumn{2}{|l|}{$\begin{array}{l}95 \% \mathrm{Cl} \\
\text { for OR }\end{array}$} \\
\hline & & & & Lower & Upper \\
\hline Univariate model difference & VFT (seconds) & 0.538 & 0.89 & 0.62 & 1.29 \\
\hline between pre- and & ARC (seconds) & 0.281 & 0.88 & 0.70 & I.II \\
\hline posttreatment & AVT (seconds) & 0.289 & 1.69 & 0.64 & 4.44 \\
\hline Multivariate model difference & $\mathrm{DRV}(\mathrm{mm})$ & 0.129 & 0.00 & 0.00 & 2.92 \\
\hline between pre- and & $\mathrm{DRA}(\mathrm{mm})$ & 0.028 & 0.00 & 0.00 & 0.01 \\
\hline \multirow[t]{6}{*}{ posttreatment } & Mean CMT $(\mu \mathrm{m})$ & 0.18 & 0.99 & 0.99 & 1.00 \\
\hline & $\mathrm{DRV}(\mathrm{mm})$ & & 0.049 & 0.00 & 0.00 \\
\hline & $\mathrm{CMT}(\mu \mathrm{m})$ & & 0.03 & 0.034 & 0.99 \\
\hline & Constant & 0.002 & 0.12 & & \\
\hline & & Group I & Group 2 & & \\
\hline & $\begin{array}{l}\text { Predicted } \\
\text { correct }\end{array}$ & $93.3 \%$ & $41.7 \%$ & & \\
\hline
\end{tabular}

Abbreviations: ARC, arm-retina circulation time; AVT, arteriovenous transit time; $\mathrm{Cl}$, confidence interval; CMT, central macular thickness; DRA, diameter of retinal artery; DRV, diameter of retinal vein; OR, odds ratio; VFT, venous filling time. 
of retinal arterioles of an average of $9 \%$ according to the pretreatment diameter was observed in the present study. Since effective vasodilatation and vasoconstriction mandate the presence of a normal capillary bed and endothelium, larger pretreatment arterioles and venular calibers and significant vasoconstriction in arterioles with treatment in patients with significant VA recovery indicate the presence of a normally functioning retinal vascular bed with maintenance of reflex responses. In neovascular AMD, CNV has been associated with ischemic changes in the choroid, and an increase in the level of ischemia correlates with AMD severity as well as overexpression of VEGF. ${ }^{18-20}$ The vascular effects of VEGF are believed to be mediated by nitric oxide (NO), which is a potent vasodilator with a major role in retinal arteriolar tone and retinal blood flow autoregulation. ${ }^{21,22}$ Hence, the identification of larger pretreatment arteriolar calibers in our patients with significant VA recovery seems to indicate greater $\mathrm{NO}$ synthesis. This greater NO synthesis may be due to greater VEGF synthesis and/or due to the NO synthesis ability of healthy endothelium. High levels of VEGF and NO cause edema through arteriolar and venous dilatation, increased blood flow, and capillary leakage. Pretreatment larger vessel diameters may indicate a higher severity of underlying AMD. ${ }^{10}$ In our study, although not statistically significant, there was a tendency for thicker pretreatment OCT values $(>50 \mu \mathrm{m})$ in patients with significant VA recovery, and a more pronounced and significant CMT decrease $(>80 \mu \mathrm{m})$ in these patients following ranibizumab treatment, which seems to have been achieved by the presence of an adapted vascular bed.

In addition, the decrease in retinal arteriolar diameter may not be a true vasoconstriction. In patients with active neovascular AMD, vasodilation may indicate upregulation of VEGF levels, and, after the treatment, VEGF blockade may provide a return to the previous retinal arteriole diameter. ${ }^{14}$ Even when VEGF is higher, the preservation of a functional retinal vascular bed with normal reflex responses seems to enable the higher functionality of sensorial retina and much better VA recovery. The contrary significant dilatation of retinal venular caliber following intravitreal injections of ranibizumab over 3 months was reported in eyes with neovascular AMD. ${ }^{13}$ Even though in our study we did not observe a significant change in the mean difference between pre- and posttreatment vein diameter, it tended to be lower in Group 1 and higher in Group 2. This may show the passive adaptation of the venules to the changes in arteriolar diameters in eyes with significant VA recovery. In the other eyes, venular dilatation could be observed due to the inability of the arterioles to change their diameters. The degree of retinal venular dilatation at diagnosis of CNV has been indicated as a possible marker of disease activity, with an increased VEGF level in the eye predicting poorer response to anti-VEGF therapy. ${ }^{10}$ In Group 2, we observed that the absence of significant arteriolar narrowing after three intravitreal injections was a predictive factor for the near-impossibility of $0.2 \log$ MAR units of recovery of VA. However, Group 2 also included eyes in which VA was preserved. CMT decrease and VA preservation may be due to a decrease in the flow rate without a significant decrease in arteriolar diameter. Further, the exact magnitude of retinal vessel diameter decrease that is accepted as clinically important is not known. In our opinion, ranibizumab injection could be repeated when needed, taking into account that VA could be protected by its use.

\section{Conclusion}

Our findings regarding the effect of the intravitreal injection of ranibizumab on retinal circulation times and vessel caliber in patients with AMD reveal that ranibizumab is associated with a decrease in retinal arteriolar diameter, a decrease in CMT, and the preservation (42\%) or significant recovery of VA (37\%) in the overall population. Notably, a more favorable VA prognosis was evident in patients with pretreatment larger retinal vessels as well as in patients with more prominent reductions in DRA and CMT after therapy. Further larger prospective studies with longer follow-up would reveal the effect of anti-VEGF treatment on retinal vasculature more clearly and would define the potency of arteriolar caliber change as a predictive factor for functional prognosis.

\section{Disclosure}

This study was first presented as an e-poster at the 12th Euretina Congress, September 6-9, 2012, Milan, Italy. The authors declare they have no proprietary or commercial interest in any materials discussed in this article. All authors state that they have no conflicts of interest in this work.

\section{References}

1. Kulkarni AD, Kuppermann BD. Wet age-related macular degeneration Adv Drug Deliv Rev. 2005;57(14):1994-2009.

2. Ng EW, Adamis AP. Targeting angiogenesis, the underlying disorder in neovascular age related macular degeneration. Can J Ophthalmol. 2005;40(3):352-368

3. Aiello LP, Avery RL, Arrigg PG, et al. Vascular endothelial growth factor in ocular fluid of patients with diabetic retinopathy and other retinal disorders. N Engl J Med. 1994;331(22):1480-1487.

4. Brown DM, Kaiser PK, Michels M, et al. Ranibizumab versus verteporfin for neovascular age-related macular degeneration. $N$ Engl J Med. 2006;355(14):1432-1444 
5. Rosenfeld PJ, Brown DM, Heier JS, et al. Ranibizumab for neovascular age-related macular degeneration. $N$ Engl J Med. 2006; 355(14):1419-1431.

6. Kourlas H, Abrams P. Ranibizumab for the treatment of neovascular age-related macular degeneration: a review. Clin Ther. 2007; 29(9):1850-1861.

7. Papadopoulou DN, Mendrinos E, Mangioris G, Donati G, Pournaras CJ. Intravitreal ranibizumab may induce retinal arteriolar vasoconstriction in patients with neovascular age-related macular degeneration. Ophthalmology. 2009;116(9):1755-1761.

8. Nguyen TT, Islam FM, Farouque HM, et al. Retinal vascular caliber and brachial flow mediated dilation: the Multi-Ethnic Study of Atherosclerosis. Stroke. 2010;41(7):1343-1348.

9. Frampton JE. Ranibizumab: a review of its use in the treatment of neovascular age-related macular degeneration. Drugs Aging. 2013; 30(5):331-358.

10. Wickremasinghe SS, Busija L, Guymer RH, Wong TY, Qureshi S. Retinal venular caliber predicts visual outcome after intravitreal ranibizumab injection treatments for neovascular AMD. Invest Ophthalmol Vis Sci. 2012;53(1):37-41.

11. Tatlipinar S, Dinç UA, Yenerel NM, Görgün E. Short-term effects of a single intravitreal bevacizumab injection on retinal vessel calibre. Clin Exp Optom. 2012;95(1):94-98.

12. Avery RL, Pieramici DJ, Rabena MD, Castellarin AA, Nasir MA, Giust MJ. Intravitreal bevacizumab (Avastin) for neovascular agerelated macular degeneration. Ophthalmology. 2006;113(3):363-372.

13. Lalwani GA, Rosenfeld PJ, Fung AE, et al. Variable-dosing regimen with intravitreal ranibizumab for neovascular age-related macular degeneration: year 2 of the PrONTO Study. Am J Ophtahlmol. 2009; 148(1):43-58.

14. Wickremasinghe SS, Guymer RH, Wong TY, Kawasaki R, Wong W, Qureshi S. Retinal venular calibre dilatation after intravitreal ranibizumab treatment for neovascular age-related macular degeneration. Clin Experiment Ophthalmol. 2012;40(1):59-66.
15. Jaffe GJ, Martin DF, Toth CA, et al. Macular morphology and visual acuity in the comparison of age-related macular degeneration treatments trials. Ophthalmology. 2013;120(9):1860-1870.

16. Boyer DS, Antoszyk AN, Awh CC, et al. Subgroup analysis of the MARINA study of ranibizumab in neovascular age-related macular degeneration. Ophthalmology. 2007;114(2):246-252.

17. Fontaine O, Olivier S, Descovich D, Cordahi G, Vaucher E, Lesk MR. The effect of intravitreal injection of bevacizumab on retinal circulation in patients with neovascular macular degeneration. Invest Ophthalmol Vis Sci. 2011;52(10):7400-7405.

18. Frank RN, Amin RH, Eliott D, Puklin JE, Abrams GW. Basic fibroblast growth factor and vascular endothelial growth factor are present in epiretinal and choroidal neovascular membranes. Am J Ophthalmol. 1996;122(3):393-403.

19. Lip PL, Blann AD, Hope-Ross M, Gibson JM, Lip GY. Age-related macular degeneration is associated with increased vascular endothelial growth factor, hemorheology and endothelial dysfunction. Ophthalmology. 2001;108(4):705-710.

20. Tilton RG, Chang KC, LeJeune WS, Stephan CC, Brock TA, Williamson JR. Role for nitric oxide in the hyperpermeability and hemodynamic changes induced by intravenous VEGF. Invest Ophthalmol Vis Sci. 1999;40(3):689-696.

21. Harris A, Ciulla TA, Chung HS, Martin B. Regulation of retinal and optic nerve blood flow. Arch Ophthalmol. 1998;116(11): 1491-1495.

22. Yokoyama K, Choshi T, Kimoto K, Shinoda K, Nakatsuka K. Retinal circulatory disturbances following intracameral injection of bevacizumab for neovascular glaucoma [letter]. Acta Ophthalmol. 2008;86(8):927-928.
Clinical Ophthalmology

\section{Publish your work in this journal}

Clinical Ophthalmology is an international, peer-reviewed journal covering all subspecialties within ophthalmology. Key topics include: Optometry; Visual science; Pharmacology and drug therapy in eye diseases; Basic Sciences; Primary and Secondary eye care; Patient Safety and Quality of Care Improvements. This journal is indexed on Submit your manuscript here: http://www.dovepress.com/clinical-ophthalmology-journal

\section{Dovepress}

PubMed Central and CAS, and is the official journal of The Society of Clinical Ophthalmology (SCO). The manuscript management system is completely online and includes a very quick and fair peer-review system, which is all easy to use. Visit http://www.dovepress.com/ testimonials.php to read real quotes from published authors. 